Supporting Information for:

\title{
Bioinspired Polymerization of Quercetin to Produce a Curcumin-loaded Nanomedicine with Potent Cytotoxicity and Cancer Targeting Potential In Vivo
}

Suhair Sunoqrot ${ }^{\mathrm{ab} *}$, Tahany Al-Debsi ${ }^{\mathrm{a}}$, Eveen Al-Shalabi ${ }^{\mathrm{a}}$, Lina Hasan ${ }^{\mathrm{a}}$, Farid Nazer Faruqu ${ }^{\mathrm{b}}$, Adam Walters ${ }^{\mathrm{b}}$, Robert Palgrave ${ }^{\mathrm{c}}$, and Khuloud T. Al-Jamal ${ }^{\mathrm{b}}$.

${ }^{a}$ Department of Pharmacy, Faculty of Pharmacy, Al-Zaytoonah University of Jordan, Amman 11733, Jordan

${ }^{b}$ Institute for Pharmaceutical Science, King's College London, London SE1 9NH, UK

${ }^{c}$ Department of Chemistry, University College London, London WC1H OAJ, UK

\section{*Corresponding Author:}

Suhair Sunoqrot, $\mathrm{PhD}$

Associate Professor of Pharmaceutics

Department of Pharmacy

Faculty of Pharmacy

Al-Zaytoonah University of Jordan

P.O. Box 130, Amman 11733, Jordan

Phone: +962-6-4291511 Ext. 197

Fax: +962-6-4291432

Email: suhair.sunoqrot@zuj.edu.jo

No. of pages: 7

No. of figures: 6 


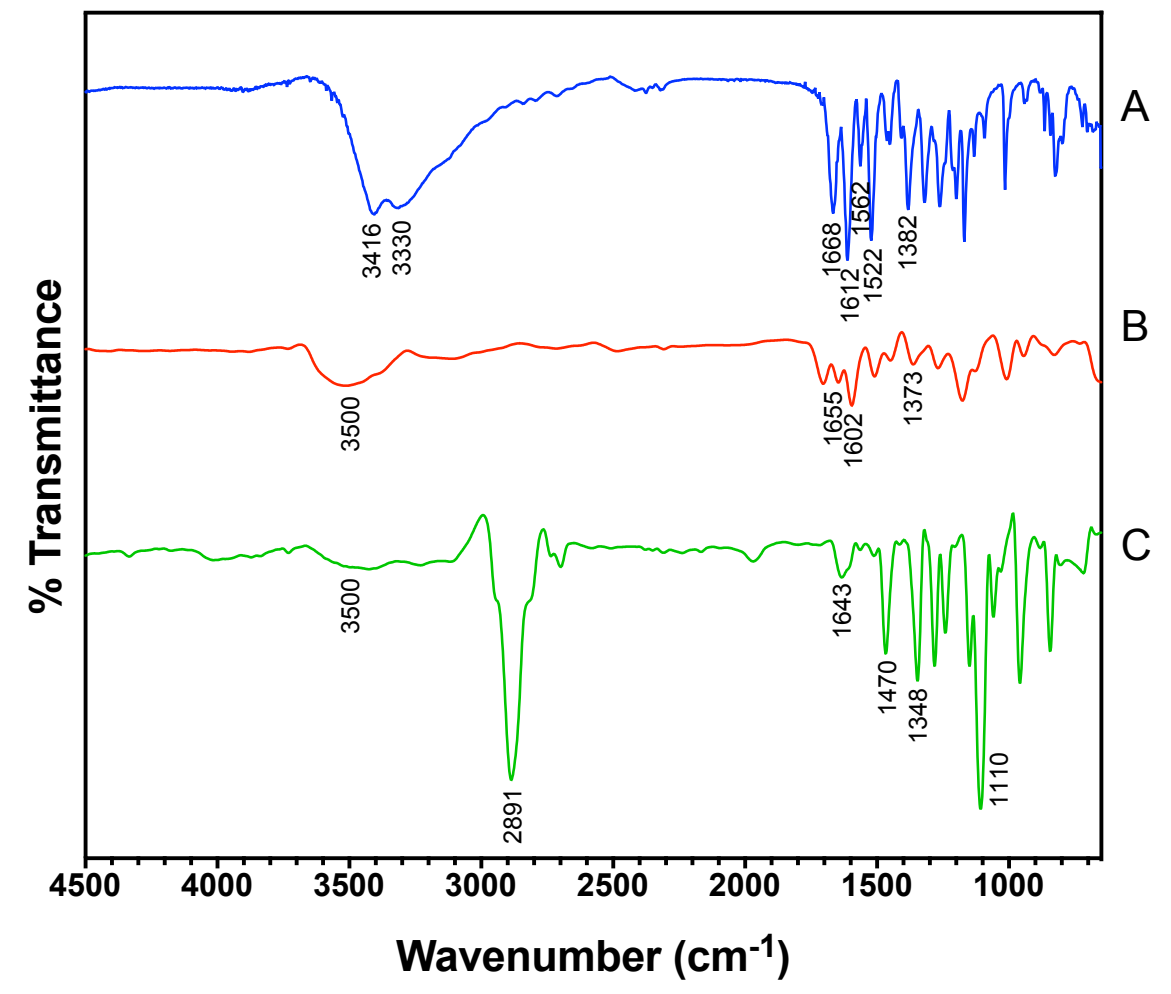

Figure S1. FT-IR spectra of (A) QCT, (B) QCT NPs, and (C) PEGylated QCT NPs (QCT@PEG NPs). 

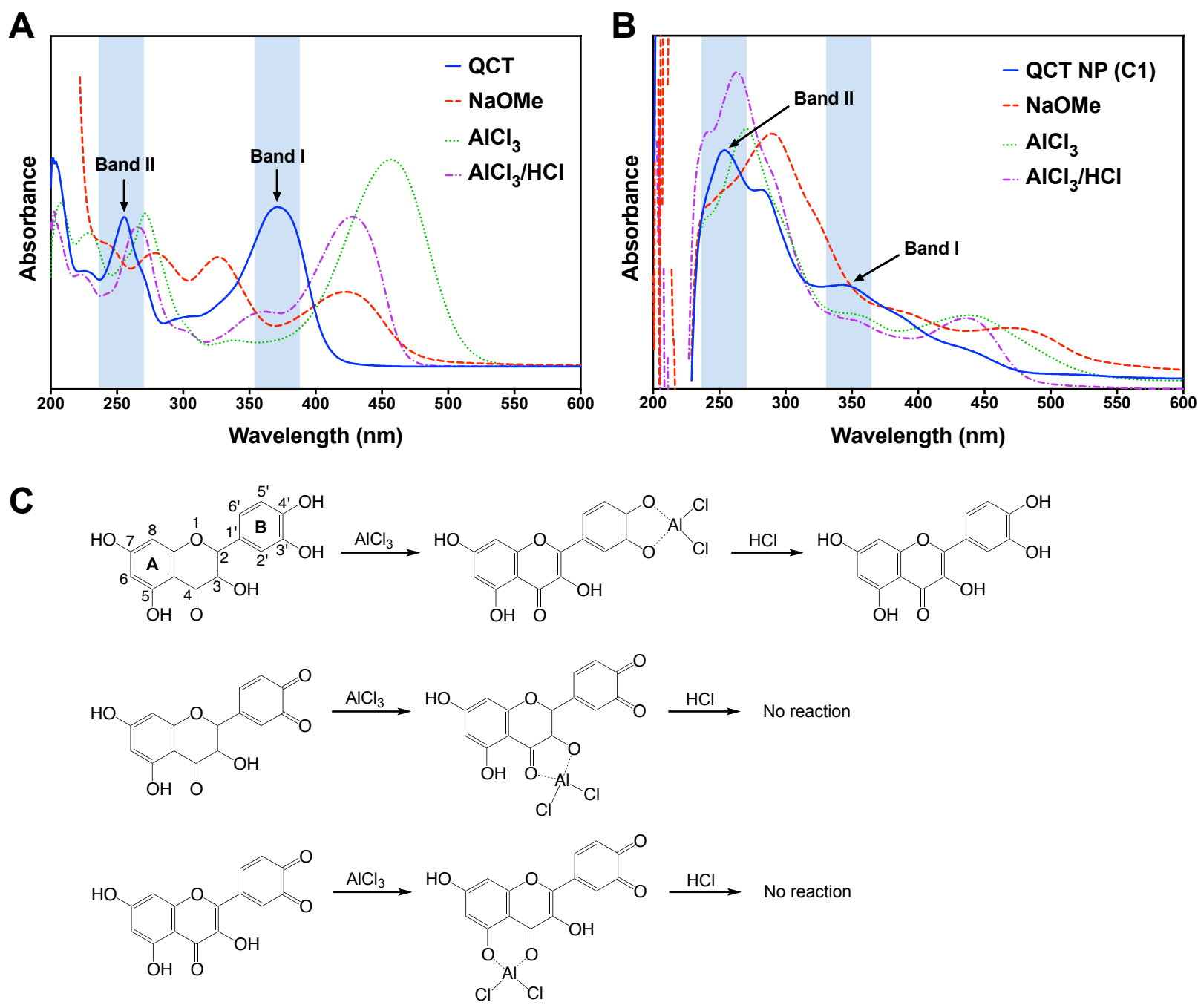

Figure S2. UV-Vis spectra of (A) QCT and (B) QCT NPs in methanol before and after the addition of $\mathrm{NaOMe}, \mathrm{AlCl}_{3}$, and $\mathrm{AlCl}_{3} / \mathrm{HCl}$; (C) Types of complexes that can form between $\mathrm{AlCl}_{3}$ and QCT depending on the oxidative state of the $o$-dihydroxyl groups on ring B. 


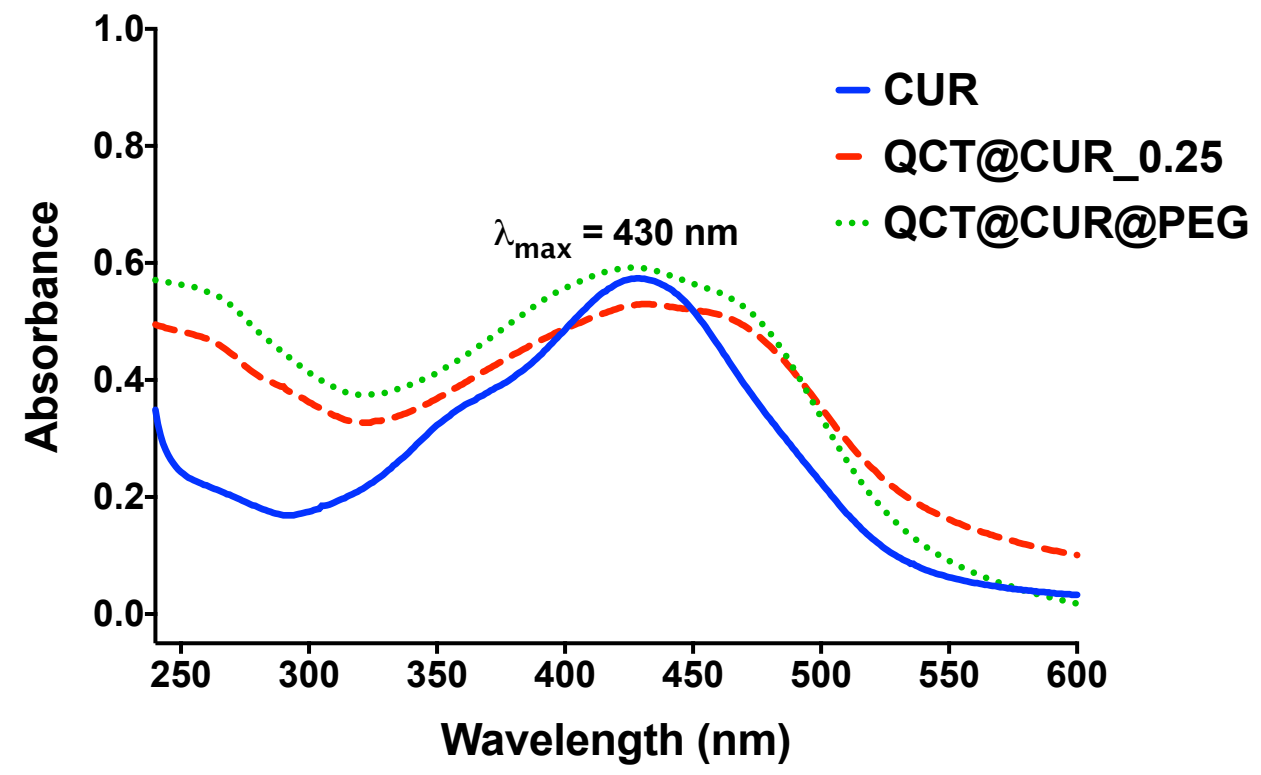

Figure S3. UV-Vis spectra of CUR (solid line), CUR-loaded QCT NPs (QCT@CUR_0.25 NPs; dashed line), and PEGylated CUR-loaded QCT NPs (QCT@CUR@PEG NPs; dotted line) in DMSO. 
A

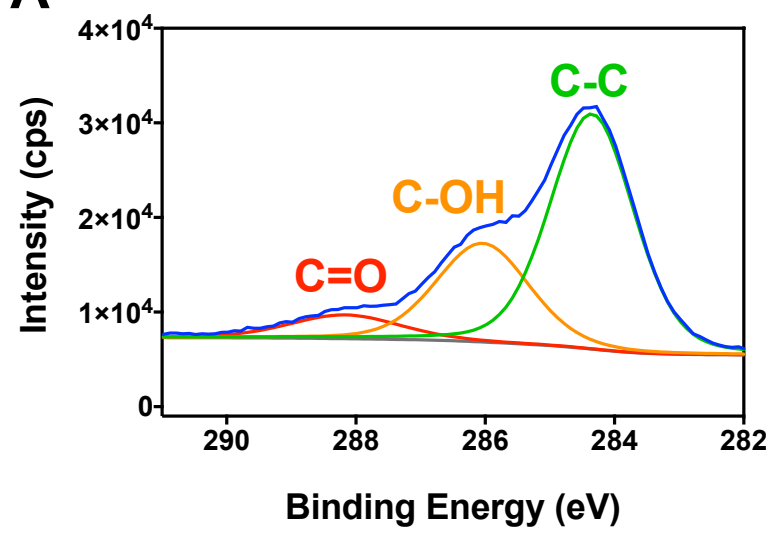

C

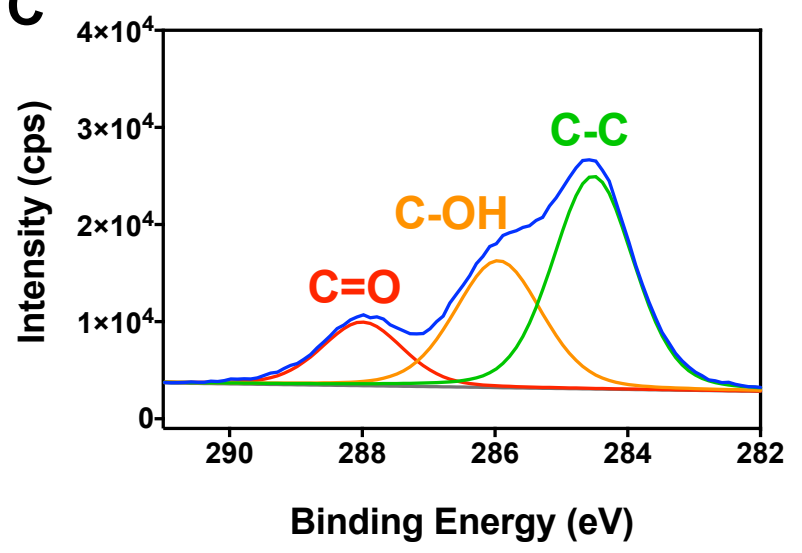

B
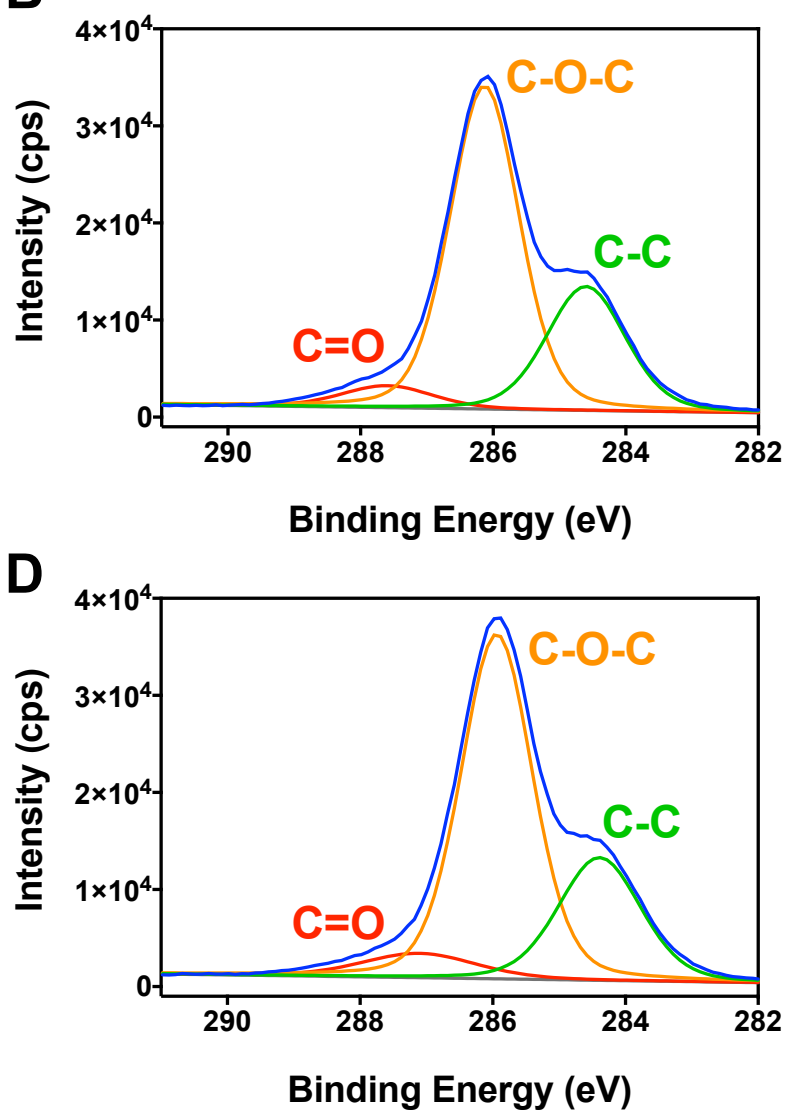

Figure S4. Deconvoluted XPS high-resolution spectra of C 1s regions of (A) QCT NPs, (B) PEGylated QCT NPs (QCT@PEG NPs), (C) CUR-loaded QCT NPs (QCT@CUR_0.25 NPs), and (D) PEGylated CUR-loaded QCT NPs (QCT@CUR@PEG NPs), with peak assignments inferred from literature values. The increase in C-O-C components observed in (B) and (D) compared to (A) and (C) is attributed to immobilization of mPEG-SH on the surface of QCT NPs. 

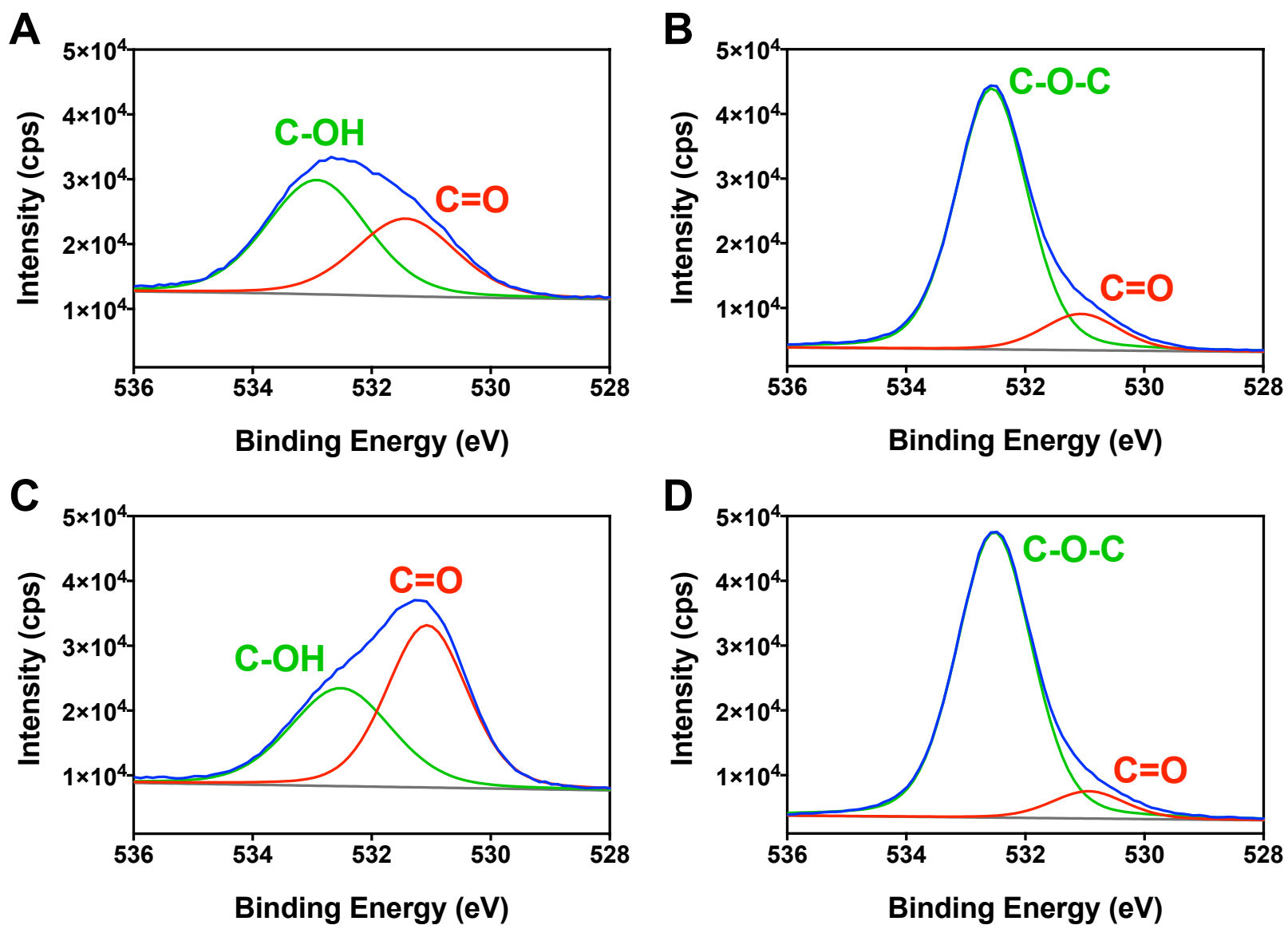

Figure S5. Deconvoluted XPS high-resolution spectra of O 1s regions of (A) QCT NPs, (B) PEGylated QCT NPs (QCT@PEG NPs), (C) CUR-loaded QCT NPs (QCT@CUR_0.25 NPs), and (D) PEGylated CUR-loaded QCT NPs (QCT@CUR@PEG NPs), with peak assignments inferred from literature values. The increase in C-O-C components observed in (B) and (D) compared to (A) and (C) is attributed to immobilization of mPEG-SH on the surface of QCT NPs. 


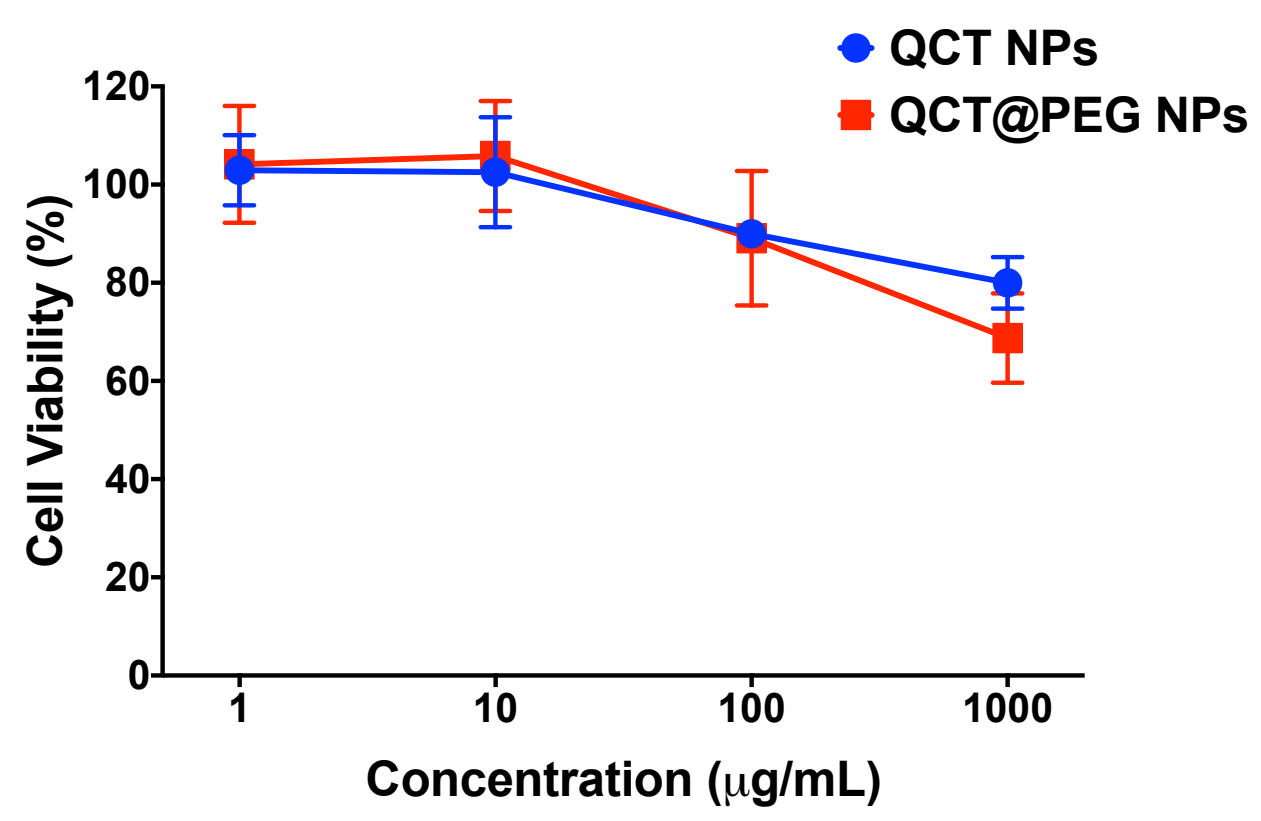

Figure S6. Cell viability of CT26 cells after $72 \mathrm{~h}$ incubation with drug-free QCT NPs and PEGylated QCT NPs (QCT@PEG NPs) (n=4) showing low cytotoxicity up to $1 \mathrm{mg} / \mathrm{mL}$. 